\title{
Human health and disease in a microbial world
}

\author{
Peter J. Turnbaugh ${ }^{1 *}$ and Alain Stintzi ${ }^{2}$ \\ 1 FAS Center for Systems Biology, Harvard University, Cambridge, MA, USA \\ 2 Department of Biochemistry, Microbiology and Immunology, Ottawa Institute of Systems Biology, University of Ottawa, Ottawa, ON, Canada \\ *Correspondence: pturnbaugh@fas.harvard.edu
}

Humans and other mammals have co-evolved with trillions of microorganisms (the microbiota), whose aggregate genomes (the microbiome) contribute functions that are not encoded by our own human genome. Recent developments in the emerging field of metagenomics have enabled an unprecedented description of organismal, genetic, transcriptional, proteomic, and metabolic diversity across a wide range of body habitats, spawning multiple ongoing international human microbiome projects. These largely sequencing-driven studies, along with mechanistic studies in vitro and in animal models, have emphasized the role the human microbiome plays in health and disease, including inflammatory bowel disease (IBD), metabolic syndrome, autoimmune disorders, and even cancer.

Here, in this "Research Topic" collection of reviews, perspectives, and original research articles, leading researchers in the field have highlighted recent developments in our appreciation of the structure and function of the human microbiome, spanning a wide range of topics including immune development (Derrien et al., 2011; Kosiewicz et al., 2011; Pfeiffer and Sonnenburg, 2011; Reading and Kasper, 2011; Smith and Garrett, 2011), IBD (Young et al., 2011), nutrition, and obesity (Geurts et al., 2011; Li et al., 2011; Lin, 2011; Thomas et al., 2011), and novel methods for analyzing complex microbial communities (Moore et al., 2011; Parfrey et al., 2011).

First, we present a series of reviews highlighting that the human immune system is intimately linked to the gut microbiome; the gut microbiota influences immune development, susceptibility to infection from pathogens, and inflammation (Kosiewicz et al., 2011; Reading and Kasper, 2011; Smith and Garrett, 2011). Through the use of gnotobiotic mouse models colonized with cultured isolates from the human gut, recent studies have highlighted the role specific members of this complex community can play in programming the immune system. For example, segmented filamentous bacteria can promote the expansion of IL-17 cells, Bacteroides fragilis can alleviate colitis in animal models, and some Clostridium strains can promote the expansion of regulatory T-cells. Moving forward, the study of gnotobiotic mice colonized with more complex communities derived from human or animal donors, may provide insight into the mechanisms by which different microbial communities can alter host immunity (Reading and Kasper, 2011). In an original research article, Derrien et al. (2011) demonstrate that Akkermansia muciniphila, a bacterium specialized for the degradation of host mucin (a glycoprotein abundant in the mucosal layer of the gut), is able to alter mucosal gene expression, up-regulating genes for immune response and cell fate determination, with potentially important implications for immune tolerance.

Together, these interactions between the gut microbiome and immune system could contribute to multiple diseases involving aberrant inflammation. The most commonly studied case, although still poorly understood, is that of IBD. Young et al. (2011) argue that IBD should be considered a cluster of related diseases, that detailed time series analysis of luminal and mucosa-associated microbial communities are required, and that a comprehensive understanding of the relevant confounding variables in human clinical studies will be necessary. Perhaps most importantly, many technical aspects must be considered in designing a given study, spanning the methods used for sampling patients, the development of appropriate animal models, the platform used for sequencing, and the nascent development of bioinformatic methods for the concomitant analysis of DNA, RNA, proteins, metabolites, and spatial organization. Of note, recent studies are beginning to reveal the diversity of microbial eukaryotes living in the human gut, a largely underexplored component of our microbiome with potential implications for health and disease (Parfrey et al., 2011).

Another key method necessary to move beyond largely descriptive sequence-based analyses of the gut microbiome is functional metagenomics; the screening of metagenomic libraries created by cloning community DNA into a suitable host strain, allowing causal links between genes from uncultured organisms and their function (Moore et al., 2011). To date, studies of the human gut and other environments have already identified novel genes for antibiotic resistance, fiber degradation, bile salt hydrolysis, and immune modulation (Moore et al., 2011).

Pfeiffer and Sonnenburg (2011) highlight the role of the gut microbiome in regulating host susceptibility to infection from pathogenic bacteria and viruses. For example, infection by Clostridium difficile and Salmonella sp. can be triggered by antibiotic treatment, while other pathogens may alter the host immune response to promote persistent colonization. Furthermore, the gut microbiome may play an important role in viral infection: e.g., influenza, dengue, adenovirus, and HIV. Future studies promise to address the underlying mechanisms behind these phenomena, such as directly modifying viral particles, recruiting immune effectors that promote viral replication, or modulating host immunity to permit viral replication (Pfeiffer and Sonnenburg, 2011).

The human microbiome is also thought to play a critical role in both sides of the energy balance equation, allowing the digestion of complex substrates inaccessible to our own human enzymes and promoting the accumulation of body fat. Intriguingly, studies in livestock suggest that antibiotics can be used to alter weight gain (Lin, 2011). Thomas et al. (2011) review their recent results highlighting the potential for a new type of impact of the diet on our gut microbiome - the lateral transfer of genes from transient microbes ingested from the diet to our resident microorganisms. These genetic swaps could potentially have a lasting impact on the ability to digest a given diet, enabling an entrenched community to adapt to changes in diet. In two original research articles published 
here, animal models are used to further explore this relationship between the gut microbiome and energy balance. Geurts et al. (2011) demonstrate that the gut microbiota and endocannabinoid system are modified in obese and diabetic leptin-resistant $d b / d b$ mice, although follow-on studies are necessary to determine if the gut microbiota is contributing to or responding to this phenotype. Finally, Li et al. (2011) use rats to show that bariatric surgery results in an altered microbiota and metabolome, coupled to increased cytotoxicity.

Together, these original research articles, reviews, and perspectives emphasize the importance of the microbiome across many aspects of human health and disease. Although many important questions remain unanswered, the pace of discovery continues to

\section{REFERENCES}

Derrien, M., Van Baarlen, P., Hooiveld, G., Norin, E., Muller, M., and De Vos, W. (2011). Modulation of mucosal immune response, tolerance and proliferation in mice colonized by the mucin-degrader Akkermansia muciniphila. Front. Microbiol. 2:166. doi: 10.3389/fmicb.2011.00166

Geurts, L., Lazarevic, V., Derrien, M., Everard, A., Van Roye, M., Knauf, C., Valet, P., Girard, M., Muccioli, G. G., Francois, P., De Vos, W. M., Schrenzel, J., Delzenne, N. M., and Cani, P. D. (2011). Altered gut microbiota and endocannabinoid system tone in obese and diabetic leptin-resistant mice: impact on apelin regulation in adipose tissue. Front. Microbiol. 2:149. doi: 10.3389/fmicb.2011.00149

Kosiewicz, M. M., Zirnheld, A. L., and Alard, P. (2011). Gut microbiota, immunity and disease: a complex relationship. Front. Microbiol. 2:180. doi: $10.3389 /$ fmicb. 2011.00180
Li, J., Reshat, R., Wu, Q., Ashrafian, H., Bueter, M., Le Roux, C., Darzi, A., Athanasiou, T., Marchesi, J., Nicholson, J., Holmes, E., and Gooderham, N. (2011). Experimental bariatric surgery in rats generates a cytotoxic chemical environment in the gut contents. Front. Microbiol. 2:183. doi: 10.3389/ fmicb.2011.00183

Lin, J. (2011). Effect of antibiotic growth promoters on intestinal microbiota in food animals: a novel model for studying the relationship between gut microbiota and human obesity? Front. Microbiol. 2:53. doi: 10.3389/ fmicb.2011.00053

Moore, A. M., Munck, C., Sommer, M. O. A., and Dantas, G. (2011). Functional metagenomic investigations of the human intestinal microbiota. Front. Microbiol. 2:188. doi: 10.3389/ fmicb. 2011.00188

Parfrey, L. W., Walters, W. A., and Knight, R. (2011). Microbial eukaryotes in the human microbiome: ecology,

accelerate, as a wide range of scientists across many disciplines are beginning to turn their expertise toward studying this complex and fascinating ecosystem. In the near future, these studies may lead to new diagnostic tools and therapies targeting our microbial communities. In a broader sense, they also provide a new perspective of our own human body, as an often imperceptible, but always ongoing, dialog with our trillions of microbial partners.

\section{ACKNOWLEDGMENTS}

We thank the Frontiers editorial staff for assistance in putting together this research topics collection. Peter J. Turnbaugh is supported by NIH P50 GM068763. Alain Stintzi is supported by CIHR.

evolution, and future directions. Front. Microbiol. 2:153. doi: 10.3389/ fmicb.2011.00153

Pfeiffer, J. K., and Sonnenburg, J. L. (2011). The intestinal microbiota and viral susceptibility. Front. Microbiol. 2:92. doi: 10.3389/fmicb.2011.00092

Reading, N. C., and Kasper, D. L. (2011). The starting lineup: key microbial players in intestinal immunity and homeostasis. Front. Microbiol. 2:148 doi: 10.3389/fmicb.2011.00148

Smith, P. M., and Garrett, W. S. (2011). The gut microbiota and mucosal T cells. Front. Microbiol. 2:111. doi: 10.3389/fmicb.2011.00111

Thomas, F., Hehemann, J. H., Rebuffet, E., Czjzek, M., and Michel, G. (2011). Environmental and gut bacteroidetes: the food connection. Front. Microbiol. 2:93. doi: 10.3389/ fmicb.2011.00093

Young, V. B., Kahn, S. A., Schmidt, T. M., and Chang, E. B. (2011). Studying the enteric microbiome in inflammatory bowel diseases: getting through the growing pains and moving forward. Front. Microbiol. 2:144. doi: 10.3389/ fmicb.2011.00144

Received: 26 August 2011; accepted: 26 August 2011; published online: 16 September 2011.

Citation: Turnbaugh PJ and Stintzi A (2011) Human health and disease in a microbial world. Front. Microbio. 2:190. doi: 10.3389/fmicb.2011.00190

This article was submitted to Frontiers in Cellular and Infection Microbiology, a specialty of Frontiers in Microbiology.

Copyright (c) 2011 Turnbaugh and Stintzi. This is an open-access article subject to a non-exclusive license between the authors and Frontiers Media SA, which permits use, distribution and reproduction in other forums, provided the original authors and source are credited and other Frontiers conditions are complied with. 